\title{
Cross-Ratios and Angles Determine a Polygon*
}

\author{
J. Snoeyink \\ Department of Computer Science, University of North Carolina, \\ Chapel Hill, NC 27599, USA \\ snoeyink@cs.unc.edu
}

\begin{abstract}
We prove that a unique simple polygon is determined, up to similarity, by the interior angles at its vertices and the cross-ratios of diagonals of any given triangulation. (The cross-ratio of a diagonal is the product of the ratio of edge lengths for the two adjacent triangles.) This establishes a conjecture of Driscoll and Vavasis, and shows the correctness of a key step of their algorithm for computing Schwarz-Christoffel transformations mapping a disk to a polygon.
\end{abstract}

\section{Introduction}

Computing meshes for the numerical solution of problems on domains with irregular geometry poses interesting challenges because it interweaves continuous mathematics with discrete geometry and then seeks an implementation on an even more discrete computer. One excellent example is a new algorithm by Driscoll and Vavasis for computing a conformal mapping of a unit disk to a simple plane polygon [2]. We describe this algorithm in some detail in Section 2. The correctness of one of its steps depends on a purely geometric conjecture [2, Conjecture 1]:

Let $P$ be an $n$-vertex, triangulated, simple polygon. Then $P$ is uniquely determined up to similarity transform by the following data: the sequence of all interior angles of $P$ at its vertices, and the list of $n-3$ absolute values of cross-ratios of the quadrilaterals determined by the triangulation of $P$.

Pairs of adjacent triangles, which share a diagonal edge of the triangulation, form the

\footnotetext{
* This research was supported in part by an NSERC Research Grant. Portions of this work were performed while the author was on leave at the Geometry Center at the Johns Hopkins University, and in project Prisme at INRIA Sophia-Antipolis.
} 


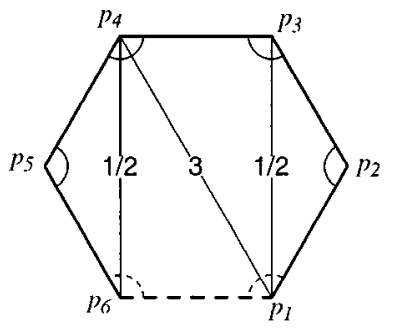

Fig. 1. Angles and cross-ratios.

above-mentioned quadrilaterals. The cross-ratio of a quadrilateral is the product of the lengths of two sides counterclockwise from the endpoints of the diagonal divided by the product of the lengths of the two sides clockwise. That is, if a quadrilateral has sides of length $a, b, c$, and $d$ in ccw order starting from one endpoint of the diagonal, we define the cross-ratio to be $a c /(b d)$. In the triangulated hexagon of Fig. 1, diagonal edges are labeled with their cross-ratios. (Note: Driscoll and Vavasis [2] use the reciprocal of this ratio.)

In this paper we establish their conjecture by establishing properties of partially specified polygons, which we define in Section 4. We employ some observations on the ratios of triangles that we make in Section 3.

\section{The Schwarz-Christoffel Formula and the CRDT Algorithm}

If we think of a simple polygon $P=\left\{p_{1}, p_{2}, \ldots, p_{n}\right\}$ as a simply connected, open subset of the complex plane and let $D$ be the unit disk, then the Riemann Mapping Theorem [3] says that there is an analytic function $f: D \rightarrow P$ mapping the unit disk to the polygon. If the point $f(0)$ and direction of $f^{\prime}(0)$ are specified, then $f$ is unique. With this mapping, some problems on $P$ (e.g., Laplace's equation) can be solved on the simpler domain $D$. Other problems that require a mesh on $P$ can begin with a mesh on $D$; conformal mapping preserves angles in the mesh.

All mappings $f: D \rightarrow P$, from the unit disk $D$ to an $n$-gon $P$, can be written using the Schwarz-Christoffel formula

$$
f(w)=p+q \int_{0}^{w} \prod_{1 \leq j \leq n}\left(1-\frac{\omega}{w_{j}}\right)^{\beta_{j}} d \omega .
$$

In this formula the complex numbers $p$ and $q$ translate, rotate, and scale the image, the scalars $\beta_{j}=\psi_{j} / \pi-1$, where $\psi_{j}$ is the interior angle at polygon vertex $p_{j}$, and the unit-length complex numbers $w_{j}$ are the prevertices, the points on the boundary of the disk that map to the vertices of the polygon. The integration is a complex contour integral in the disk. The $\mathrm{SC}$ formula has $n+4$ parameters. Eliminating similarity transformations of the polygon and the freedom to choose $f(0)$ and the direction of its derivative leaves $n-3$ parameters unspecified. These are determined by numerically solving a difficult nonlinear system [1], [2], [4], [5]. 
Attempts to compute the prevertices and use the resulting SC formula over the entire polygon $P$ suffer from a phenomenon called crowding - skinny regions of $P$ cause the angle between the prevertices to decrease exponentially in the aspect ratio. Crowding causes problems for numerical techniques that compute prevertices and use them in the $\mathrm{SC}$ formula; many bits of precision can be lost when determining the angle between two prevertices.

Driscoll and Vavasis [2] have developed an clever combination of numerical and geometric ideas to minimize the impact of crowding. They use a constrained Delaunay triangulation of $P$, with new vertices introduced on the boundary so that triangles in $P$ have good aspect ratios. There is a corresponding triangulation of prevertices in $D$ (join prevertices iff an edge joins their vertices in $P$ ); Driscoll and Vavasis reformulate the nonlinear system to compute the cross-ratios of this triangulation. These $n-3$ crossratios are three parameters too few to specify the $n$ prevertices completely. They note that when an application is concerned with accuracy of computations inside a particular triangle, it can specify the three prevertices of this triangle to be spread out around the disk, then determine the rest of the prevertices from the cross-ratios in linear time. Crowding increases further from the specified triangle, but usually the importance of a vertex decreases with distance as well.

As an illustration of their technique, they produced the right side of Fig. 2, which shows a mapping from a rectangle to a "maze" polygon. Solid lines in the rectangle at $i / 5$, for $1 \leq i \leq 4$, and dotted lines at $10^{-2}, 10^{-4}, \ldots, 10^{-16}$ map to their images at right. Driscoll and Vavasis [2] report that "all computations were performed in double precision."

While the experimental evidence supports the correctness and efficiency of their approach, it does depend on some conjectures. The first, cited in Section 1 and established in this paper, relates to their iteration to find prevertices by finding the cross-ratios of the triangulation of prevertices. Their iteration uses the SC formula to compute crossratios of $P$ from the guessed cross-ratios of prevertices; the question is whether, having determined the cross-ratios of polygon $P$, the algorithm has determined $P$. There are other interesting conjectures about the relationship between the magnitudes of crossratios in $P$ and in $D$, which we cannot yet address.

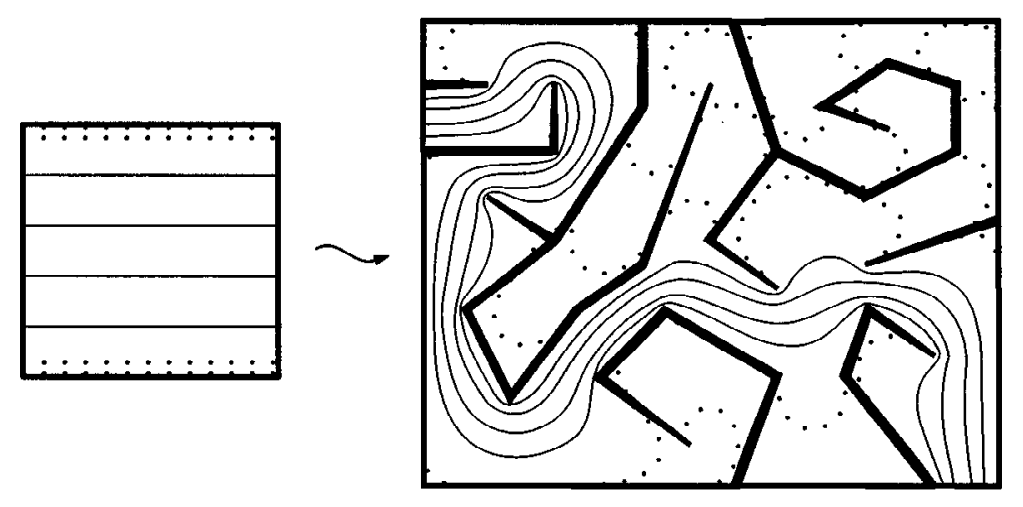

Fig. 2. Conformal mapping from rectangle to maze. (From [2].) 


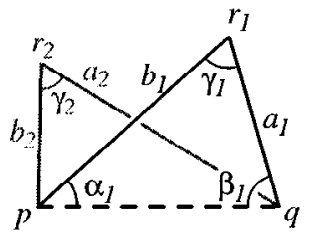

Fig. 3. Triangle notation.

\section{Observations on the Ratios of Triangles}

To begin, we establish several sufficient conditions on sides and angles of a triangle for its ratio to increase. We use the notation illustrated in Fig. 3: Points $p$ and $q$ are fixed so that the segment $\overline{p q}$ is horizontal from left to right. Points $r_{1}$ and $r_{2}$ are chosen above the line $\overleftrightarrow{p q}$, and the angles of triangle $p q r_{i}$ measure $\alpha_{i}, \beta_{i}$, and $\gamma_{i}$ at $p, q$, and $r_{i}$, respectively. Let $a_{i}$ and $b_{i}$ denote the lengths of $q r_{i}$ and $p r_{i}$, respectively. The ratio of the triangle $p q r_{i}$ will thus be $a_{i} / b_{i}$.

If we fix $\gamma_{1}=\gamma_{2}$, then the triangle is determined when $\alpha_{i}$ is chosen. Thus, we establish the next condition by a continuous argument and drop the subscripts.

Lemma 1. Using the above notation, when angle $\gamma$ is fixed, the ratio $r(\alpha)=a / b$ is $a$ strictly increasing function of $\alpha$, for $\alpha \in[0, \pi-\gamma]$.

Proof. By the law of sines, the ratio

$$
r(\alpha)=\frac{a}{b}=\frac{\sin \alpha}{\sin (\alpha+\gamma)}=\cos \gamma-\sin \gamma \cdot \cot (\alpha+\gamma)
$$

This is an invertible function for $0<\alpha \leq \pi-\gamma$, and its derivative $\sin \gamma / \sin ^{2}(\alpha+\gamma)$ is positive for $0<\gamma<\pi$, which we must have for $p q r$ to be a triangle.

By the way, all of our conditions are established by algebraic arguments, which is a good way to build character. To develop intuition, it is sometimes more pleasant to observe the conditions geometrically by considering the interaction of four families of constraint curves that cover the plane, which are illustrated in Fig. 4. We use these to illustrate the algebraic proofs.

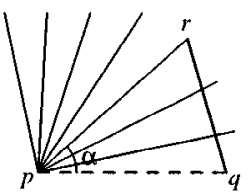

fixed angle $\alpha$

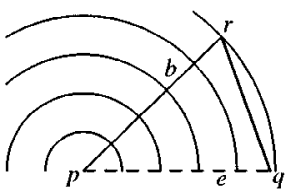

fixed ratio $b / e$

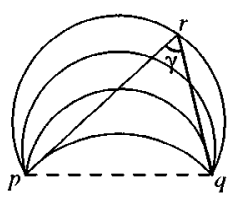

fixed angle $\gamma$

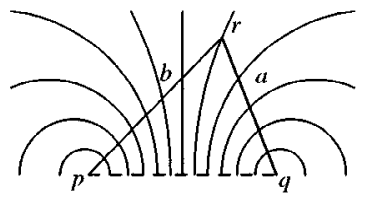

fixed ratio $a b$

Fig. 4. Four families of constraint curves for triangle endpoint $r$. 


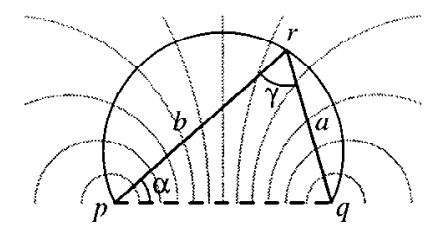

Fig. 5. Fixed $\gamma$.

Observation 2. Let pqr be a triangle with $p$ and $q$ fixed and $r$ to the left of $\overline{p q}$. The following families are the loci of points $r$ with the corresponding parameter of triangle pqr fixed:
A. Points with fixed angle $\alpha=\angle r p q$ lie on the lines through $p$.
B. Points with fixed ratio $b / e=r p / p q$ lie on the circles centered at $p$.
C. Points with fixed angle $\gamma=\angle q$ rp lie on circles through $p$ and $q$.
D. Points with fixed ratio $a / b=q r / r p$ lie on circles orthogonal to those of $C$.

These curves intersect nicely; a pair of curves chosen from A and B, A and C, B and $\mathrm{D}$, or from $\mathrm{C}$ and $\mathrm{D}$ will have only one intersection above the $x$ axis. Families $\mathrm{A}$ and $\mathrm{B}$ and families $\mathrm{C}$ and $\mathrm{D}$ are orthogonal—any two of their curves intersect at right angles.

A geometric argument for Lemma 1 observes that with angle $\gamma$ fixed, the point $r$ moves along a circular arc of family $\mathrm{C}$ as $\alpha_{i}$ increases, see Fig. 5. As $r$ moves, it crosses each curve of family $\mathrm{D}$, and the ratio increases over $(0, \infty)$.

Lemma 3. Using the triangle notation of Fig. 3, the ratios $a_{1} / b_{1}<a_{2} / b_{2}$ if $r_{1} \neq r_{2}$ and any of the four following conditions hold:

(1) if $a_{1} \leq a_{2}$ and $b_{1} \geq b_{2}$,

(2) if $b_{1} \geq b_{2}, \alpha_{1} \leq \alpha_{2}$, and $\gamma_{1} \geq \gamma_{2}$,

(3) if $a_{1} \leq a_{2}, \beta_{1} \geq \beta_{2}$, and $\gamma_{1} \leq \gamma_{2}$, or

(4) if $a_{1} \geq a_{2}, b_{1} \leq b_{2}, \alpha_{1} \leq \alpha_{2}$, and $\beta_{1} \geq \beta_{2}$.

Proof. Condition (1) is the easiest. With the assumption that $r_{1} \neq r_{2}$, one of the inequalities $a_{1} \leq a_{2}$ or $b_{1} \geq b_{2}$ must be strict, so when condition (1) holds, $a_{1} / b_{1}<$ $a_{2} / b_{2}$.

For the remaining conditions, it helps to express ratios of sides using the law of sines and trigonometric identities. For example,

$$
\frac{a_{i}}{b_{i}}=\frac{\sin \alpha_{i}}{\sin \beta_{i}}=\frac{\sin \left(\gamma_{i}+\beta_{i}\right)}{\sin \beta_{i}}=\cos \gamma_{i}+\cot \beta_{i} \sin \gamma_{i}=\cos \gamma_{i}+\cos \beta_{i}\left(\frac{\|q-p\|}{b_{i}}\right) .
$$

Note that all angles will be in $(0, \pi)$, so sines are positive. Cosine and cotangent are decreasing functions over this angle range, crossing zero at angle $\pi / 2$.

When condition (2) holds we consider two cases, depending on whether angle $\beta_{1}$ is obtuse. 


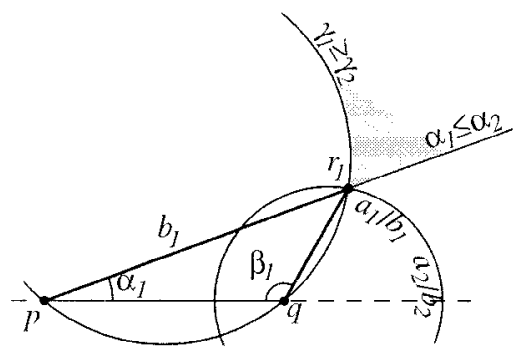

Fig. 6. $\beta_{1}$ obtuse.

Case 1: $\beta_{1}$ obtuse (Fig. 6). Assume, for the sake of deriving a contradiction, that $a_{1} / b_{1} \geq a_{2} / b_{2}$, or equivalently

$$
\cos \gamma_{1}+\cot \beta_{1} \sin \gamma_{1} \geq \cos \gamma_{2}+\cot \beta_{2} \sin \gamma_{2} \text {. }
$$

Since $\beta_{1}>\pi / 2$, the value $-\cot \beta_{1}$ is positive. Also, $\pi / 2>\gamma_{1} \geq \gamma_{2}$, so $\cos \gamma_{1} \leq \cos \gamma_{2}$ and $\sin \gamma_{1} \geq \sin \gamma_{2}$. Combining these inequalities, we observe that $-\cot \beta_{1} \leq-\cot \beta_{2}$. We conclude that $\pi_{2}<\beta_{1} \leq \beta_{2}$ and $\alpha_{1} \leq \alpha_{2}<\pi / 2$.

However, then $\sin \alpha_{1} \leq \sin \alpha_{2}$ and $\sin \beta_{1} \geq \sin \beta_{2}$, and, if $r_{1} \neq r_{2}$, one of these inequalities is strict. Thus we derive a contradiction,

$$
\frac{a_{1}}{b_{1}}=\frac{\sin \alpha_{1}}{\sin \beta_{1}}<\frac{\sin \alpha_{2}}{\sin \beta_{2}}=\frac{a_{2}}{b_{2}} .
$$

Case 2: $\beta_{1}$ not obtuse (Fig. 7). Assume, for the sake of deriving a contradiction, that $a_{1} / b_{1} \geq a_{2} / b_{2}$. Let $e$ denote the fixed length of the edge from $p$ to $q$. The condition $\alpha_{1} \leq \alpha_{2}$ implies $\cos \alpha_{1} \geq \cos \alpha_{2}$. By the condition $b_{1} \geq b_{2}$, we know

$$
\cos \alpha_{1}+\cos \beta_{1}\left(\frac{a_{1}}{b_{1}}\right)=\frac{e}{b_{1}} \leq \frac{e}{b_{2}}=\cos \alpha_{2}+\cos \beta_{2}\left(\frac{a_{2}}{b_{2}}\right) \text {. }
$$

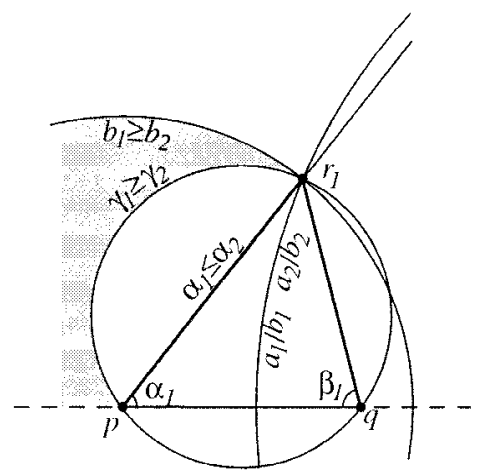

Fig. 7. $\beta_{1}$ not obtuse. 


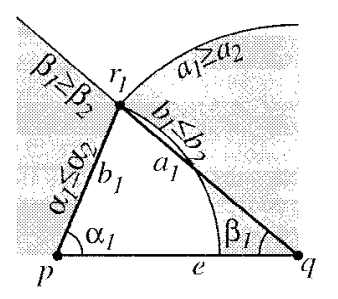

Fig. 8. Angle and distance constraints.

Since $\cos \beta_{1}$ is nonnegative, we can combine the above inequalities to derive that $0 \leq$ $\cos \beta_{1} \leq \cos \beta_{2}$. However, since $\cos \gamma_{1} \leq \cos \gamma_{2}$ and $e / b_{1} \leq e / b_{2}$, with one of these inequalities strict if $r_{1} \neq r_{2}$, we again derive a contradiction:

$$
\frac{a_{1}}{b_{1}}=\cos \gamma_{1}+\cos \beta_{1}\left(\frac{e}{b_{1}}\right)<\cos \gamma_{2}+\cos \beta_{2}\left(\frac{e}{b_{2}}\right)=\frac{a_{2}}{b_{2}} .
$$

We reduce condition (3) to condition (2). Reflect the triangle about a vertical line, which exchanges $a$ 's with $b$ 's, and $\alpha$ 's with $\beta$ 's. Then exchange 1's with 2's, and apply the proof for condition (2). When we return the conclusion to the original notation, we obtain that $b_{2} / a_{2}<b_{1} / a_{1}$. Taking reciprocals gives the desired result.

The final part of the lemma is vacuously true-condition (4) cannot be satisfied unless $r_{1}=r_{2}$.

By the law of sines $b_{i}=e \sin \beta_{i} / \sin \gamma_{i}=e \cos \alpha_{i}+a_{i} \cos \gamma_{i}$, where $e=\|q-p\|$ is fixed (see Fig. 8). Since $\cos \alpha_{1} \geq \cos \alpha_{2}$ and $a_{1} \geq a_{2}$, in order to have $b_{1} \leq b_{2}$ (and $r_{1} \neq r_{2}$ ), we must either have $0 \leq \cos \gamma_{1}<\cos \gamma_{2}$ or $\cos \gamma_{1}<0$. Similarly, $a_{i}=e \cos \beta_{i}+b_{i} \cos \gamma_{i}, \cos \beta_{1} \leq \cos \beta_{2}$, and $b_{1} \leq b_{2}$. In order to have $a_{1} \geq a_{2}$ (and $r_{1} \neq r_{2}$ ), we must either have $0 \leq \cos \gamma_{2}<\cos \gamma_{1}$ or $\cos \gamma_{2}<0$. Because the inequalities with nonnegative cosines are incompatible, we must have that both $\gamma_{1}$ and $\gamma_{2}$ are obtuse angles.

We can now observe a contradiction: angles $\alpha_{i}$ and $\beta_{i}$ are not obtuse, so $\sin \alpha_{1} \leq \sin \alpha_{2}$ and $\sin \beta_{1} \geq \sin \beta_{2}$. By the law of sines, $a_{i} / b_{i}=\sin \alpha_{i} / \sin \beta_{i}$, but

$$
\frac{a_{1}}{b_{1}} \leq \frac{a_{2}}{b_{2}} \quad \text { and } \quad \frac{\sin \alpha_{1}}{\sin \beta_{1}} \geq \frac{\sin \alpha_{2}}{\sin \beta_{2}}
$$

Only if $r_{1}=r_{2}$ can these inequalities be satisfied simultaneously.

\section{A Proof Using Partial Polygons}

The remainder of this paper proves that the interior angles and cross-ratios of a triangulated polygon $P$ are sufficient to determine $P$ up to similarity. The proof uses partial polygons, for which all cross-ratios and all angles except the first and last are specified. In notation, let $P=p_{1} p_{2} \cdots p_{n}$ be a simple polygon in the Euclidean plane. Assume 


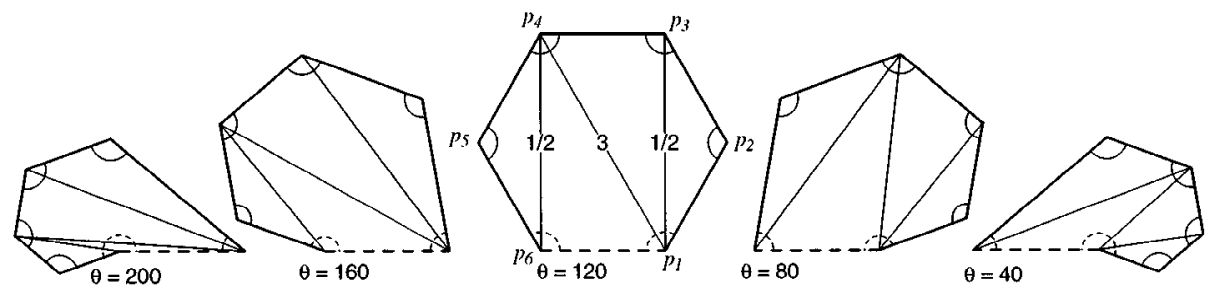

Fig. 9. Realizations of a partial polygon $P$ for five different angles $\theta$. In each, the angles at $p_{2}$ through $p_{5}$ are $120^{\circ}$, and cross-ratios are $1 / 2,3$, and $1 / 2$ as labeled in the middle hexagon.

that we are given:

- $n-2$ angles $\psi_{2}, \psi_{3}, \ldots, \psi_{n-1}$, where $0<\psi_{i} \leq 2 \pi$ measures the internal angle at $p_{i}$,

- $n-3$ cross-ratios for the quadrilaterals formed by the pairs of triangles adjacent to the $n-3$ diagonals in the triangulation of $P$.

Note that angles $\psi_{1}$ and $\psi_{n}$ at vertices $p_{1}$ and $p_{n}$ are not specified. We call $\psi_{n}$ the variable angle of partial polygon $P$. If we set $\psi_{n}=\theta$, we can determine $\psi_{1}$ since the sum of interior angles $\sum_{1 \leq i \leq n} \psi_{i}=(n-2) \pi$.

A realization of partial polygon $P$ is an embedding that respects the specified angles and cross-ratios and has positive, finite area for all triangles; Fig. 9 shows five realizations of a triangulated hexagon for different values of $\theta$. No global monotonicity properties for realizations of $P$ seem to be evident as $\theta$ varies-e.g., the area and sides of triangle $p_{1} p_{2} p_{3}$ shrink and grow again as $\theta$ decreases in Fig. 9.

We call $\overline{p_{n} p_{1}}$ the unspecified edge of partial polygon $P$. It is one side of a triangle in the triangulation of $P$; let $a$ and $b$ be the lengths of the other two sides in counterclockwise order, and define the ratio of $P$ to be $a / b$. We prove by induction that the ratio of $P$ is a partial function of $\theta$ that is strictly increasing over its domain.

Theorem 4. Let $P$ be a partial polygon with variable angle $\theta$. For each $\theta$, the realization of $P$, if it exists, is unique, and the ratio of $P$ is a strictly increasing partial function $r(\theta)$.

Proof. We prove this by induction on the number of triangles in $P$.

In the base case, partial polygon $P$ is a single triangle $p_{1} p_{2} p_{3}$. We can choose the positions of $p_{1}$ and $p_{3}$ as in Fig. 10 to fix the similarity transformation. Since angle $\psi_{2}$ at $p_{2}$ is specified, Lemma 1 applies to show that the ratio is a strictly increasing function of the variable angle $\theta$ at $p_{3}$.

In the induction step, we are given a partial polygon $P$, having $n>3$ vertices, $n-2$ triangles, and variable angle $\theta$ and unspecified edge $e$, which we fix as horizontal. Removing the triangle incident on the unspecified edge leaves a pair of partial polygons or, when this triangle uses vertex $p_{n-1}$ or $p_{2}$, a single partial polygon. We handle these cases separately. 


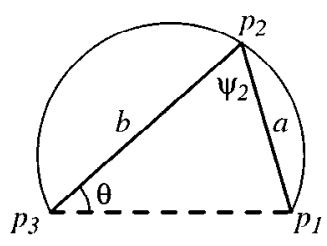

Fig. 10. Base case.

Single using $p_{n-1}$. When triangle $p_{1} p_{n-1} p_{n}$ is adjacent to the unspecified edge $e$ of $P$, then removing this triangle leaves a partial polygon $P^{\prime}$ from $p_{1}$ to $p_{n-1}$ to which we may apply the induction hypothesis.

We define notation as in Fig. 11: Let $\varphi$ be the variable angle for $P^{\prime}$, and let $a$ be the length of the unspecified edge of $P^{\prime}$. Let $\gamma=\psi_{n-1}-\varphi$. Let $b$ be the length of $\overline{p_{n-1} p_{n}}$, which is the third side of the removed triangle, and let $\beta$ be the angle opposite the side of length $b$. Let $\kappa$ be the cross-ratio specified for diagonal $\overline{p_{n-1} p_{1}}$. Define the cross-ratio function $s(\varphi)$ to be the ratio for $P^{\prime}$ divided by $\kappa$. By the induction hypothesis, $s(\varphi)$ is a strictly increasing partial function.

We want to prove that the ratio $r(\theta)=a / b$ is a strictly increasing partial function. First, we note that a given angle $\theta$ determines at most one realization of $P$. As $\varphi$ varies from 0 to $\psi_{n-1}$, the function $s(\varphi)$ increases. At the same time, the angle $\gamma=\psi_{n-1}-\varphi$ decreases; for a fixed $\theta$, Lemma 1 says that the ratio $e / b$ also decreases. Because any realization of $P$ has $e / b=s(\varphi)$, the realization for any fixed $\theta$ is unique.

Now, to show that $r(\theta)=a / b$ is strictly increasing, we compare the ratios for two realizations of $P$ with two different angles, $\theta_{1}<\theta_{2}$. We consider two subcases, depending on whether the corresponding angles $\gamma_{1} \geq \gamma_{2}$ or $\gamma_{1}<\gamma_{2}$. (As with the lemmas of the previous section, we can assume that $e$ remains fixed, and subscript other lengths and angles in the two realizations.)

If $\gamma_{1} \geq \gamma_{2}$, then $\varphi_{1} \leq \varphi_{2}$, so the ratio $e / b_{1}=s\left(\varphi_{1}\right) \leq s\left(\varphi_{2}\right)=e / b_{2}$. Since angles $\theta_{1}<\theta_{2}$ correspond to the $\alpha$ 's of Lemma 3, we have the conditions of part (2) and can conclude that the ratio increases: $r\left(\theta_{1}\right)=a_{1} / b_{1}<a_{2} / b_{2}=r\left(\theta_{2}\right)$.

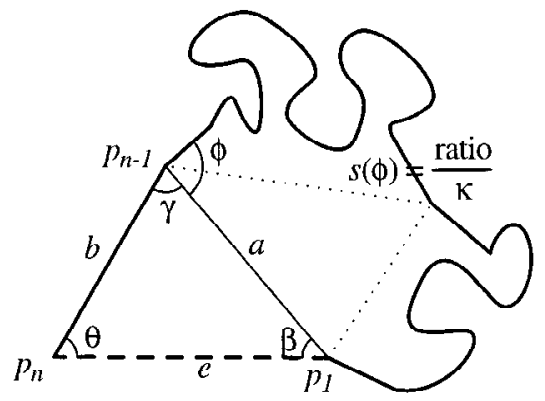

Fig. 11. Single partial polygon. 
On the other hand, if $\gamma_{1} \leq \gamma_{2}$, then $\varphi_{1} \geq \varphi_{2}$, so the ratio $e / b_{1}=s\left(\varphi_{1}\right) \geq s\left(\varphi_{2}\right)=e / b_{2}$. Since the angles of the triangle sum to $\pi$, we know that $\beta_{1} \geq \beta_{2}$. Thus, when $a_{1} \leq a_{2}$ we can apply Lemma 3(3), and when $a_{1} \geq a_{2}$ we apply Lemma 3(4); in either case, the ratio increases: $r\left(\theta_{1}\right)=a_{1} / b_{1}<a_{2} / b_{2}=r\left(\theta_{2}\right)$.

This completes the argument for the single case using $p_{n-1}$.

Single using $p_{2}$. When triangle $p_{1} p_{2} p_{n}$ is adjacent to the unspecified edge of $P$, we can reduce this case to the previous. Reflect $P$ about a line, taking the reciprocals of all specified cross-ratios, to obtain $P_{R}$. Apply the argument of the previous case to show that there is a strictly increasing function of angle $\theta_{R}$ for the ratio $r_{R}\left(\theta_{R}\right)$ of $P_{R}$. Then there is a strictly increasing function of angle $\theta$ for the ratio $r(\theta)$ of $P$, namely

$$
r(\theta)=\frac{1}{r_{R}\left((n-2) \pi-\left(\sum_{2 \leq i \leq n-1} \psi_{i}\right)-\theta\right)} .
$$

Uniqueness is preserved under reflection.

Pair using $p_{j}$. When triangle $p_{1} p_{j} p_{n}$ is adjacent to the unspecified edge $e$ of $P$, for some $2<j<n-1$, we can label the sides $a=\overline{p_{1} p_{j}}$ and $b=\overline{p_{j} p_{n}}$, and angles $\alpha, \beta$, and $\gamma=\pi-\alpha-\beta$ opposite the sides $a, b$, and $e$. We fix the similarity by choosing edge $e$ to be horizontal as in Fig. 12.

Removing triangle abe leaves two partial polygons: one from $p_{j}$ to $p_{n}$ with variable angle $\varphi=\theta-\alpha$ and cross-ratio function $s(\varphi)$, and another from $p_{1}$ to $p_{j}$ with variable angle $\chi$ and cross-ratio function $t(\chi)$. (Because the angle at $p_{j}$ is specified as $\psi_{j}$, and the angles of a $k$-gon sum to $(k-2) \pi$, we know that $\chi+\gamma-\varphi$ is a constant, namely $(n-j-1) \pi-\sum_{j \leq k<n} \psi_{k}$. Similarly, $\theta+\beta-\chi$ is a constant.) We can apply the induction hypothesis to both partial polygons, so the cross-ratio functions $s(\varphi)$ and $t(\chi)$ are strictly increasing partial functions of their angles.

We begin with a different decomposition of $P$, however, to show that the realization of $P$ for a given $\theta$ is unique. Break $P$ into two partial polygons at edge $a$. If we fix $\theta$, then the induction hypothesis applies to the polygon from $p_{j}, \ldots, p_{n}, p_{1}$ : the ratio $b / e$ is a strictly increasing partial function of variable angle $\beta$, and its reciprocal $e / b$ is decreasing. With $\theta+\beta-\chi$ a constant and $\theta$ fixed, however, angle $\chi$ and ratio function $t(\chi)$ are also strictly increasing (partial) functions of $\beta$, and $t(\chi)=e / b$. Equality holds at most once for any specified $\theta$.

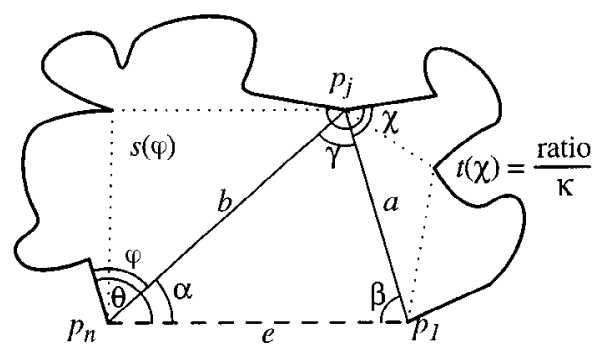

Fig. 12. Pair of partial polygons. 
Now, to show that $r(\theta)=a / b$ is strictly increasing, we compare the ratios for two realizations of $P$ with two different angles, $\theta_{1}<\theta_{2}$, and consider four subcases depending on the comparisons of corresponding angles $\varphi_{1}$ with $\varphi_{2}$ and $\chi_{1}$ with $\chi_{2}$. (As before, we assume that $e$ remains fixed, and subscript other lengths and angles in the two realizations.)

Case 1: $\varphi_{1}<\varphi_{2}$ and $\chi_{1} \leq \chi_{2}$. Since $s\left(\varphi_{1}\right)=a_{1} / e<s\left(\varphi_{2}\right)=a_{2} / e$ and $t\left(\chi_{1}\right)=$ $e / b_{1} \leq t\left(\chi_{2}\right)=e / b_{2}$, the ratio $r\left(\theta_{1}\right)=a_{1} / b_{1}<a_{2} / b_{2}=r\left(\theta_{2}\right)$ increases in this case. Note that this is Lemma 3(1), but it is easier to observe directly than by paging back to the lemma.

Case 2: $\varphi_{1} \geq \varphi_{2}$ and $\chi_{1} \leq \chi_{2}$. Here, $t\left(\chi_{1}\right)=e / b_{1} \leq t\left(\chi_{2}\right)=e / b_{2}$, so $b_{1} \geq b_{2}$. Since $\alpha_{i}=\theta_{i}-\varphi_{i}$, angles $\alpha_{1}<\alpha_{2}$, and since $\gamma_{i}+\chi_{i}-\varphi_{i}$ is a constant, $\gamma_{1} \geq \gamma_{2}$. This satisfies the conditions for Lemma 3(2), so $r\left(\theta_{1}\right)=a_{1} / b_{1}<a_{2} / b_{2}=r\left(\theta_{2}\right)$ in this case, also.

Case 3: $\varphi_{1}<\varphi_{2}$ and $\chi_{1}>\chi_{2}$. Here, $s\left(\varphi_{1}\right)=a_{1} / e<s\left(\varphi_{2}\right)=a_{2} / e$. The angles $\gamma_{1}<\gamma_{2}$, since $\gamma_{i}+\chi_{i}-\varphi_{i}$ is a constant, and $\beta_{1}>\beta_{2}$, since $\beta_{i}+\theta_{i}-\chi_{i}$ is a constant. This satisfies the conditions for Lemma 3(3), so $r\left(\theta_{1}\right)=a_{1} / b_{1}<a_{2} / b_{2}=r\left(\theta_{2}\right)$.

Case 4: $\varphi_{1} \geq \varphi_{2}$ and $\chi_{1}>\chi_{2}$. Now, $s\left(\varphi_{1}\right)=a_{1} / e \geq s\left(\varphi_{2}\right)=a_{2} / e$ and $t\left(\chi_{1}\right)=$ $e / b_{1} \geq t\left(\chi_{2}\right)=e / b_{2}$. Angles $\alpha_{1}<\alpha_{2}$ and $\beta_{1}>\beta_{2}$, since $\alpha_{i}=\theta_{i}-\varphi_{i}$ and $\beta_{i}+\theta_{i}-\chi_{i}$ is a constant. This satisfies the conditions for Lemma 3(4), so $r\left(\theta_{1}\right)=a_{1} / b_{1}<a_{2} / b_{2}=$ $r\left(\theta_{2}\right)$ - vacuously, in fact.

This completes the pair cases; the theorem is established by mathematical induction.

Because any fully specified polygon can be made into a partial polygon by omitting the first and last internal angles, we have an immediate corollary.

Corollary 5. A triangulated n-gon $P$ is uniquely determined, up to similarity, by its sequence of internal angles and the cross-ratios of its diagonals.

\section{Open Questions}

We have proved that angles and cross-ratios determine a unique simple polygon by establishing that a partial function $r(\theta)$ is strictly increasing. It is natural to ask further questions about the behavior of this function.

There are examples in which the domain of the ratio function $r(\theta)$ is not a single interval. One such is a pentagon $p_{1} \cdots p_{5}$ with diagonals $(1,3)$ and $(1,4)$ having unit cross-ratios, angles $\varphi_{2}=\pi / 4, \varphi_{3}=\pi / 2, \varphi_{4}=3 \pi / 8$, and variable angle $\varphi_{5}=\theta$. Rather than proving this, we give the intuition from the "Single $p_{n}$ " case of the induction step. The reader may wish to refer back to Fig. 11 for the notation. Figure 13 shows three types of curves that may contain the loci for vertices $p_{n}$ satisfying $e / b=s(\varphi)$, depending on whether the ratio function $s(\varphi)$ takes on values greater, less, or both greater and less than unity. If the ratio function attains a minimum value $0<s\left(\varphi_{\min }\right)<1$, then some lines 


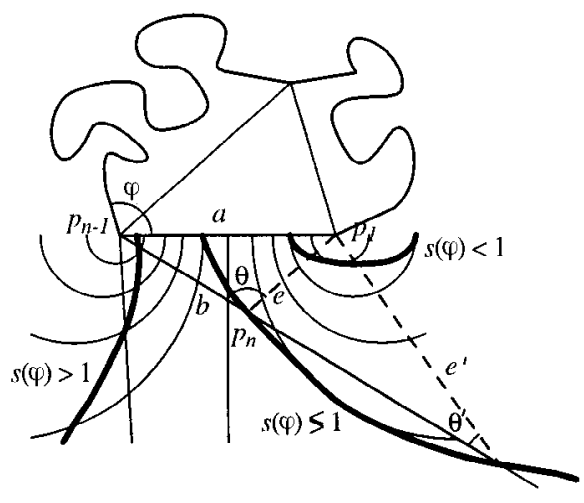

Fig. 13. Curves for $p_{n}$.

intersect this locus in two points, which correspond to two different triangles with the same ratios. We can fix the angle $\psi_{n-1}$ to be less than $\varphi_{\min }+\sin ^{-1}\left(s\left(\varphi_{\min }\right)\right)$ so that there are two solutions for $\theta$. Both solutions are preserved as $\varphi \geq \varphi_{0}$ increases, at least until the cross-ratio function becomes greater than unity. However, since $\varphi_{0}$ is minimum, angle $\varphi$ cannot decrease, and there is a portion of the curve whose angles $\theta$ do not correspond to polygon realizations.

Note that as long as the ratio attains a nonzero minimum, we can adjust the crossratio of $p_{1} p_{n-1}$ to make the minimum $s(\varphi)$ less than unity. Complete exploration of the realizations of even simple cases seems to require long sessions with a symbolic algebra package like Mathematica.

It might be nice to extract an algorithm to compute the ratio functions, and thus compute realizations, from the proof of the previous section. This not only seems difficult, but an exact implementation would require expensive, high-precision arithmetic. The CRDT algorithm of Driscoll and Vavasis [2] performs an iterative computation to find a Schwarz-Christoffel mapping of a disk to a polygon; our theorem shows that their algorithm does produce a mapping to the correct polygon.

Many interesting questions about the CRDT algorithm remain, including how to analyze its rate of convergence and quantify the worst-case crowding. It would be good to consider other interesting combinations of continuous and combinatorial geometry that address precision questions.

\section{Acknowledgments}

Marshall Bern posed this problem at the Mount Holyoke workshop on Discrete and Computational Geometry in July 1996. Günter Rote provided encouragement and suggestions to change my initial geometric proof into algebra, and Bob Thurman provided counterbalancing suggestions and encouragement. I thank Marshall, Günter, Bob, Steve Vavasis, and Jim Varah for discussions on this problem, proof, and the CRDT algorithm, and thank the referees for their comments. 


\section{References}

1. T. A. Driscoll. A MATLAB toolbox for Schwarz-Christoffel mapping. ACM Transactions on Mathematical Software, 22:168-186, 1996.

2. T. A. Driscoll and S. A. Vavasis. Numerical conformal mapping using cross-ratios and Delaunay triangulation. SIAM Journal on Scientific Computing, 19(6):1783-1803, 1998.

3. P. Henrici. Applied and Computational Complex Analysis, volume 1. Wiley, New York, 1974.

4. L. H. Howell and L. N. Trefethen. A modified Schwarz-Christoffel transformation for elongated regions. SIAM Journal of Scientific and Statistical Computing, 11(5):928-949, 1990.

5. L. N. Trefethen. Numerical computation of the Schwarz-Christoffel transformation. SIAM Journal of Scientific and Statistical Computing, 1(1):82-102, 1980.

Received April 10, 1998, and in revised form March 24, 1999. 